«Системні технології» 5 (130) 2020 «System technologies»

DOI 10.34185/1562-9945-5-130-2020-06

УДК 622.028:620.173.24

Л.М. Васильев, Д.Л. Васильев, Н.Г. Малич, А.Е Назаров

\title{
МОДЕЛИРОВАНИЕ ПРОЦЕССА РАЗРУШЕНИЯ ЦИЛИНДРИЧЕСКИХ ОБРАЗЦОВ ГОРНЫХ ПОРОД
}

Аннотация. Разработана математическая модель разрушения цилиндрических образцов горных пород при наличии контактного трения. Разработан метод расчета предельного вертикального напряжения в вершине трещины и расчета предела прочности цилиндрических образцов горных пород с использованием трех показателей свойств (предел сопротивления материала сдвигу, угол внутреннего трения, коэффициент контактного трения), которые простыми способами могут быть установлены экспериментально в условиях горнорудных предприятий, где результаты расчета могут быть оперативно использованы для управления процессами дезинтеграции.

Ключевые слова: математическая модель разрушения, цилиндрические образцы горных пород, трещина, внутреннее трение, контактное трение.

Постановка проблемы и цель исследования. Для объяснения механизма разрушения горных пород широко применяется критерий Кулона максимальных эффективных касательных напряжений на линиях скольжения (ЛС). Теория Кулона [1-3] для связных сред основана на предположении, что сопротивляемость породы сдвигу на рассматриваемой наклонной площадке равна сумме сопротивления чистому сдвигу. и величины, пропорциональной нормальному напряжению на этой площадке, от внутреннего трения

$$
\left|\tau_{\alpha}\right|=k_{n}+\mu \sigma_{\alpha}
$$

или

$$
\tau_{e} \geq k_{n}=\left|\tau_{\alpha}\right|-\mu \sigma_{\alpha}
$$

где $\tau_{\mathrm{e}}$ - эффективные касательные напряжения на линиях скольжения, Па; $\mathrm{k}_{п}$ - предел прочности материала на сдвиг; $\mu$ - коэффициент внутреннего трения; $\tau_{\alpha}$ - сопротивляемость породы сдвигу, Па; $\sigma_{\alpha}-$ нормальное напряжение на наклонной площадке, Па.

(c) Васильев Л.М., Васильев Д.Л., Малич Н.Г., Назаров А.Е, 2020 
«Системні технології» 5 (130) 2020 «System technologies»

Из первого равенства следует, что разрушение не произойдет, если левая часть меньше правой. Для определения предела прочности пород $\sigma$ (Па) на основании критерия при условии прямолинейности развития трещины - линии скольжения (ЛС) получена известная аналитическая формула $[1,2,4-6]$

$$
\sigma=\frac{2 k_{n} \cos \rho}{1-\sin \rho},
$$

где $\rho$ - угол внутреннего трения, рад.

Согласно формуле (3) предел прочности материала является постоянной величиной для любой точки на ЛС. При условии постоянства предельных вертикальных напряжений при равенстве $\tau_{e}=k_{n}$ (образование трещины) на траектории максимальных эффективных касательных напряжений (ТМЭКН) с появлением первой трещины происходило бы спонтанное разрушение тела. В реальных условиях это не происходит. Слоистые горные породы при наличии трещин несут определенную нагрузку [5]. Дело в том, что в процессе разрушения неизбежно участвует контактное трение и линии скольжения искривляются. При разрушении хрупких горных пород, обладающих внутренним и внешним трением, формула (3) существенно искажает конечный результат расчета напряжений в вершине трещины в образцах горных пород, да в целом, и в массиве. При наличии же контактных касательных напряжений, например от контактного трения, критерий Кулона предельного состояния соблюдается только локально, в вершине трещины. В остальных областях на ЛС материал находится в упругом состоянии, т.е. при $k<k_{n}$, где $k-$ текущее значение сопротивления материала сдвигу на ЛС, Па.

Поэтому для слоистых материалов формула (3) неприменима. Это положение требует разработки нового подхода к описанию процесса локального разрушения слоистых горных пород. Должны быть приняты другие исходные условия, приближающие теорию к реальным процессам. Нужно в математической модели процесса учесть локальность разрушения материала.

Цель данной статьи - разработать математическую модель и вскрыть механизм локального разрушения горных пород . 
«Системні технології» 5 (130) 2020 «System technologies»

Методика исследований. Известно, что при деформировании сплошных твердых тел образуется два вида линий скольжения. При превышении эффективными касательными напряжениями предельного значения сопротивления материала сдвигу $k_{n}$ в какой-нибудь точке на ТМЭКН происходит образование трещины. При увеличении вертикального напряжения на части материала, не вышедшей из-под нагрузки, трещина получает развитие. Для иллюстрации изобразим образец породы в виде тела с выпуклыми боковыми поверхностями, нагруженного плитами пресса при наличии контактного трения между ними и образцом с распределением контактных напряжений по Прандтлю (рис. 1). Выпуклость возникает при продольном деформировании образца за счет удержания смещения верхних слоев материала у контактных поверхностей силами контактного трения, направленными против их поперечного скольжения [3]. Знаки касательных напряжений приняты согласно известным правилам знаков.

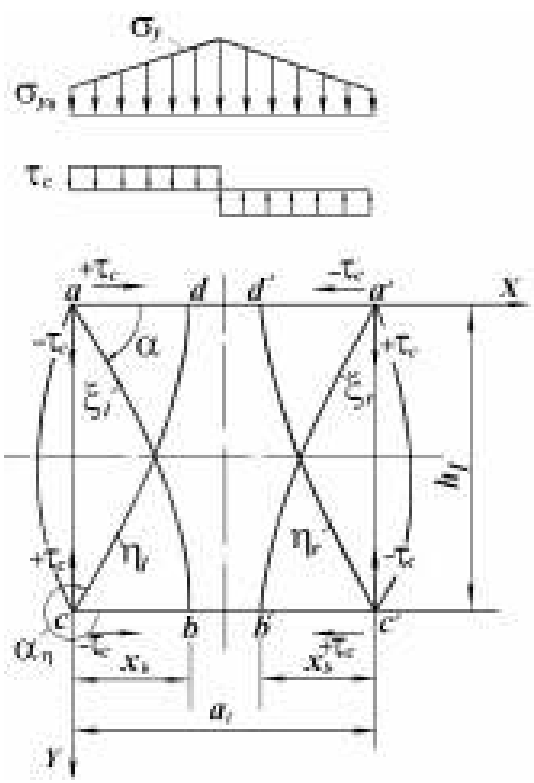

Рисунок 1 - Схемы внешних нагрузок и траекторий эффективных

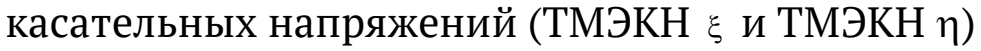
в левой и правой части образца

На рис. 1 индексами $\sigma_{y 0}, \sigma_{y}$ и $\tau_{c}$ обозначены контактные угловые, текущие нормальные и касательные напряжения, Па; $\xi_{l}, \xi_{r} \eta_{l}$ и $\eta_{r}$ - левые и правые ТМЭКН соответственно; $a_{1}$ и $h_{1}-$ длина (м) и высота (м) образISSN 1562-9945 (Print) 
«Системні технології» 5 (130) 2020 «System technologies»

ца; точки $a, a^{\prime}, c c^{\prime}$ и $b, b^{\prime}, d, d^{\prime}$ - начальные и конечные точки ТМЭКН $\xi$ и ТМЭКН $\eta$ соответственно; $\alpha_{\tau}$ и $\alpha_{\eta}{ }^{\prime}-$ углы наклона ТМЭКН $\xi$ и ТМЭКН $\eta$ относительно оси абсцисс $a X$, рад; $x_{b}$ - абсцисса точки $b\left(b^{\prime}\right)$ на нижней плоскости, м.

Для разработки метода расчета предельных напряжений используем известный метод расчета напряжений на косых площадках.

Дифференциальное уравнение равновесного состояния материала на ТМЭКН $\xi$ будет иметь вид:

$$
\frac{d \sigma_{\alpha}}{d \alpha_{\xi}}=-2\left(k+\mu \sigma_{\alpha}\right) .
$$

Аналогично поступим с выражениями напряжений на левой ТМЭКН ๆ. Получим уравнение изменения нормального напряжения со знаком плюс

$$
\frac{d \sigma_{\alpha}}{d \alpha_{\eta}}=2\left(k+\mu \sigma_{\alpha}\right),
$$

где $\alpha_{\eta}$ - угол наклона касательной к ТМЭКН п относительно оси $a X$, рад;

Решение уравнения (5) сводится к интегралу на ТМЭКН $\xi$ между точкой в вершине трещины и точкой $b$ (рис. 1)

$$
\int_{\sigma_{\alpha_{\xi}}}^{\sigma_{\alpha_{b}}} \frac{d\left(k+\mu \sigma_{\alpha_{b}}\right)}{\mu\left(k+\mu \sigma_{\alpha_{\xi}}\right)}=-2 \int_{\alpha_{\xi}}^{\alpha_{b}} d \alpha,
$$

где $\sigma_{\alpha \xi}$ и $\sigma_{\alpha_{b}}$ - нормальные напряжения в вершине трещины и в точке $b$, Па;

$\alpha_{\xi}$ и $\alpha_{b}$ - углы наклона касательной к ТМЭКН $\xi$ в вершине трещины и в точке $b$ относительно оси абсцисс $a X$, рад.

Решение уравнения (6) имеет вид

$$
\left.\ln \left(k+\mu \sigma_{\alpha}\right)\right|_{\sigma_{\alpha_{\xi}}} ^{\sigma_{\alpha b}}=-2 \mu \alpha_{b \xi},
$$

где $\alpha_{b \xi}-$ разность углов поворота ТМЭКН $\xi$ в точке $b$ и в вершине трещины, рад.

Учтем, что в вершине трещины локально на ТМЭКН $\xi$ текущее значение сопротивления породы чистому сдвигу (эффективное касательное напряжение) равно пределу прочности на сдвиг $\mathrm{k}_{\mathrm{n}}$, a в точке $b$ эффективное касательное напряжение не достигает значения предела 50 
прочности на сдвиг и обозначено через $\tau_{\mathrm{b}}$. Различие значений эффективных касательных напряжений обусловлено их зависимостью от угла поворота траекторий.

Тогда окончательно получим

$$
\frac{k_{b}+\mu \sigma_{\alpha_{b}}}{k_{n}+\mu \sigma_{\alpha \xi}}=\exp \left(-2 \mu \alpha_{b \xi}\right),
$$

где $k_{b}$ - эффективное касательное напряжение на ТМЭКН $\xi$ в контактной точке $b$, , Па.

Приведем полученную на основании решения интеграла (8) окончательную систему уравнений для определения вертикальных напряжений в вершине трещины $\sigma_{\xi(\eta)}($ Па) на ТМЭКН $\xi$ и ТМЭКН $\eta$ [3].

$$
\sigma_{y_{\xi(\eta)}}=\frac{1}{\mu}\left(\frac{k_{n}(1+\mu d)\left(1+\sin \rho \sqrt{1-b_{\xi(\eta)}^{2}}\right) \cdot \exp \left(\mp \mu\left(\beta_{b(d)}+\beta_{\xi(\eta)}\right)\right)}{1-\sin \rho \sqrt{1-b_{b(d)}^{2}}}-k_{b(d)}\right),
$$

где

$$
\begin{aligned}
& k_{b(d)}=\frac{\left(k_{n}+\mu \sigma_{y \xi(\eta)}\right) \cdot\left(1-\sin \rho \sqrt{1-b_{\xi(\eta)}^{2}}\right)}{(1+\mu d)\left(1+\sin \rho \sqrt{1-b_{b(d)}^{2}}\right) \cdot \exp \left(\mp 4 \mu \beta_{b(d)}\right)} ; \\
& d=\frac{1}{2} \arcsin \left(\frac{f_{\kappa} \sigma_{y \xi(\eta)}\left(1-\frac{2 y}{h_{1}}\right)}{k_{n}+\mu \sigma_{y \xi(\eta)}}\right) . \\
& b_{\xi(\eta)}= \pm \frac{f_{c} \cdot \sigma_{y_{\xi}(\eta)}\left(1-\frac{2 y}{h_{1}}\right)}{k_{n}+\mu \sigma_{y \xi(\eta)}} \\
& b_{b(d)}=-\frac{f_{c} \sigma_{y_{\xi(\eta)}}\left(1+\frac{x_{b(d)}}{h_{1}}\right)}{k_{b}+\mu \sigma_{y_{\xi(\eta)}}\left(1+\frac{x_{b(d)}}{h_{1}}\right)} ; \\
& \beta_{\xi(\eta)}-\frac{1}{2} \operatorname{arctg} \frac{b_{\xi(\eta)} \cos \rho}{\sin \rho-\sqrt{1-b_{\xi(\eta)}^{2}}} ; \\
& \beta_{b(d)}=-\frac{1}{2} \operatorname{arctg} \frac{b_{b(d)} \cos \rho}{\sin \rho-\sqrt{1-b_{b(d)}^{2}}} \text {. }
\end{aligned}
$$




\section{«Системні технології» 5 (130) 2020 «System technologies»}

где $d$ - коэффициент приращения прочности от действия горизонтальных напряжений,. $x_{b(d)}$ - абсцисса точки выхода ТМЭКН $\xi(\eta)$ на контактную плоскость, м; $k_{b(d)}-$ эффективные касательные напряжения на ТМЭКН $\xi$ в контактной точке $b$ (на ТМЭКН $\eta$ в точке $d$ ), Па; $b_{\xi(\eta)}-$ коэффициент в вершине трещины на ТМЭКН $\xi$ (на ТМЭКН $\eta) ; b_{b(d)}-$ коэффициент в контактной точке $b$ (на ТМЭКН $\eta$ в точке $d$ ); $\beta_{\xi(\eta)}-$ угол поворота ТМЭКН $\xi$ (ТМЭКН ๆ) от контактного трения в вершине трещины, рад; $\beta_{b(d)}$ - угол поворота ТМЭКН $\xi$ (ТМЭКН $\eta$ ) от контактного трения в точке $b(d)$, рад; $\rho-$ угол внутреннего трения, рад.

Углы наклона ТМЭКН $\xi$ и ТМЭКН ๆ относительно оси абсцисс $a X$, рад:

$$
\begin{aligned}
& \alpha_{\xi}=\frac{\pi}{4}+\frac{\rho}{2}-\beta_{\xi}, \\
& \alpha_{\eta}=\frac{7 \pi}{4}-\frac{\rho}{2}-\beta_{\eta} .
\end{aligned}
$$

где $\beta_{\xi}$ - угол поворота ТМЭКН $\xi$ от контактного трения в вершине трещины, $\beta_{\eta}$ - угол поворота ТМЭКН $\eta$ от контактного трения в вершине трещины

Результаты и их обсуждение. В качестве примера приведем расчет предела прочности цилиндрического образца хрупкой горной породы. Для расчета бу мы имеем систему из 8 уравнений при 12 параметрах, из которых три экспериментальные характеристики породы $\left(k_{n}, \mu, f_{c}\right)$ и два измеряемых геометрических параметра (длина $a_{1}$, высота $h_{1}$ ). В частном рассматриваемом случае принимаем $a_{1}=h_{1}$. В локальной области в вершине трещины, например в точке $a$ или $c$, эффективные касательные напряжения в момент разрушения по Кулону $\tau_{e}=k_{n}$. Подчеркиваем, что в других точках, вне вершины трещины, на ТМЭКН $\xi$ и ТМЭКН $\eta$ материал находится в упругом состоянии, т.е. в этих точках $\tau_{e}<k_{n}$,.

Для расчета предела прочности образца еще нужно знать распределение нормальных напряжений на контактной поверхности. Если представить нагружение на цилиндрический образец в виде двухскатной крыши согласно рис.1, то можем записать, что сила одноосного сжи- 
«Системні технології» 5 (130) 2020 «System technologies»

мающего действия на образец определяется по формуле согласно Л. Прандтлю[3]

$$
P=2 \sigma_{\xi} \int_{0}^{S_{1}}\left(1+\frac{2 f_{c} \cdot S_{1}}{2 r h_{1}}\right) d S_{1}=2 \sigma_{y_{1}}\left(S_{1}+\frac{f_{c}}{2 r h_{1}} S_{1}^{2}\right),
$$

где $S_{1}$ - половина площади поперечного сечения образца - площади сегмента круга цилиндра, $\mathbf{m}^{2}$.

Тогда предел прочности цилиндрического образца (Па):

$$
\sigma=\frac{\sigma_{y}}{S}\left(2 S_{1}+\frac{f_{c}}{r h} S_{1}^{2}\right)=\sigma_{y}\left(1+\frac{f_{c} S}{4 r h_{1}}\right),
$$

где $S$ - площадь поперечного сечения (круга) образа, м² $^{2}$

Напишем формулу контура (окружности) сечения образца (рис. 2)

$$
\left(x_{1}-r\right)^{2}+y_{1}^{2}=r^{2} \text {, }
$$

где $r$ - радиус окружности, м;

$y_{1}$ - значение ординаты точки на окружности, м;

$x_{1}$ - значение абсциссы точки на окружности, м;

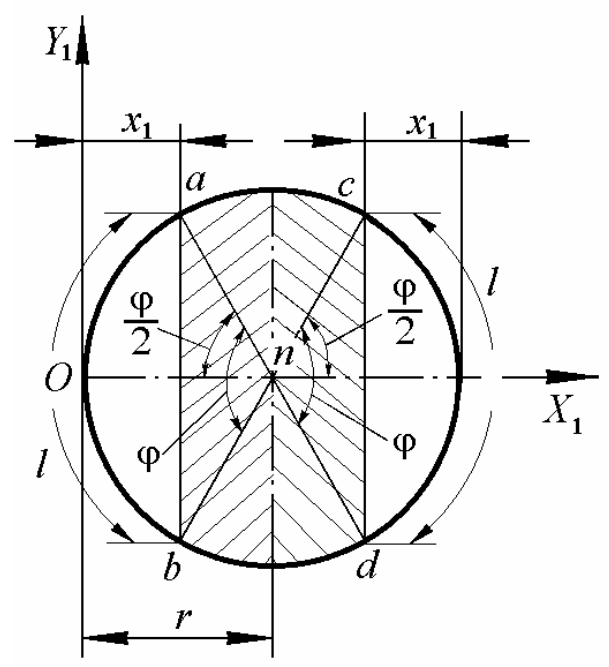

Рисунок 2 - Схема формирования несущей площади при развитии двух симметричных трещин в цилиндрическом образце

На рис. 2 обозначены: $n$ - центр круга образца; $O Y_{1}$ и $O X_{1}-$ оси системы координат; $\varphi$ - угол сегмента, рад; $l$ - длина дуги, м; $x_{1}$ - длина стрелы сегмента, м; $a, b, c$ и $d$ - точки на окружности, ограничивающие длину хорд. 
«Системні технології» 5 (130) 2020 «System technologies»

Из преобразования формулы $\left(15\right.$ - 19) имеем, $y_{1}=\sqrt{2 r x_{1}-x_{1}^{2}}$, где $y_{1}-$ ордината сегмента .

Длина хорды равна удвоенному значению $y_{1}$. Известно, что площадь сегмента

$$
S_{1}=\frac{1}{2}\left(\operatorname{lr}-2\left(\sqrt{2 r x_{1}-x_{1}^{2}}\right)\left(r-x_{1}\right)\right)
$$

где $l=2 \pi r \varphi$.

Угол ф сегмента определяется из условия, что

$$
\sin \frac{\varphi}{2}=\frac{\sqrt{2 r x_{1}-x_{1}^{2}}}{r} .
$$

Тогда имеем

$$
\varphi=\arcsin \frac{2 \sqrt{2 r x_{1}-x_{1}^{2}}}{r} .
$$

Угол сегмента, равного площади половины круга керна, равен $\pi$.

Теперь перейдем к образцу, диаметр и высота которого по ГОСТу [8] равна 0,04 м.

С использованием выражений (19) - (23) преобразуем формулу (22) и получим формулу для расчета предела прочности образца в размерности МПа при образовании первой трещины для $f_{\mathrm{c}}=0,25$

$$
\sigma=\sigma_{y}\left(1+\frac{f_{c}}{4 r h} S\right)=1,097 \cdot \sigma_{y} .
$$

Средняя сходимость расчетных значений пределов прочности с экспериментальными составляет 85,5 \%, что свидетельствует о высокой сходимости согласно известным рекомендациям А.И. Барона $[8,9]$ по допустимой достоверности теории с экспериментом и высокой результативности предлагаемого метода расчета предела прочности цилиндрических образцов горных пород. 
«Системні технології» 5 (130) 2020 «System technologies»

Таблица 1

Сопоставление расчетных значений пределов прочности при усеченно-пирамидальной форме разрушения образцов с экспериментальными данными

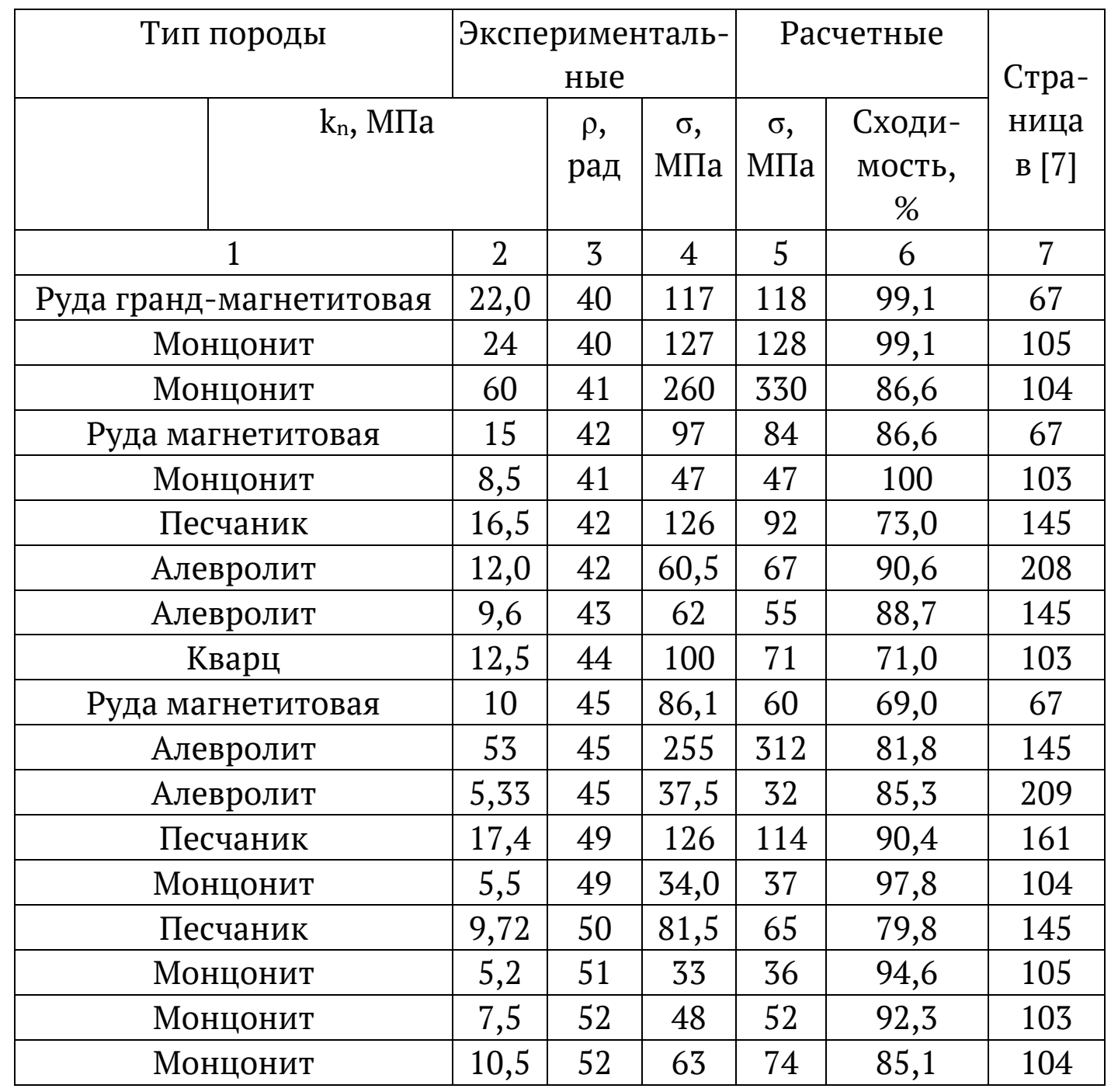

Выводы

1. Известная, широко применяемая формула предельного состояния, полученная на основании критерии прочности Кулона, не отражает процессы локального разрушения слоистых горных пород.

2. Предложена математическая модель и раскрыт механизм локального разрушения горных пород, заключающейся в формировании в вершинах трещин предела прочности сдвигу пород - предельных эффективных касательных напряжений и значения эффективных касательных 


\section{«Системні технологіï» 5 (130) 2020 «System technologies»}

напряжений, не достигающих предела прочности сдвигу в точках выхода их траекторий на граничную поверхность.

3. Выведена формула для расчета предела прочности цилиндрических образцов горных пород при усеченно-пирамидальной форме их разрушения с использованием трех показателей их свойств: $k_{n}$ - предела сопротивления материала сдвигу, $\rho$ - угла внутреннего трения, $f_{c}-$ коэффициента контактного трения, которые простыми способами могут быть установлены экспериментально в условиях горных предприятий, где результаты расчета могут быть оперативно использованы для повышения эффективности дезинтеграции.

4. Сопоставление расчетных значений пределов прочности при одноосном сжатии образцов с экспериментальными убедительно свидетельствуют о высокой результативности предлагаемого аналитического метода расчета предела прочности образцов горных пород. Средняя сходимость расчетных данных с экспериментальными составляет 85,5 \%.

\section{ЛИТЕРАТУРА / ЛІТЕРАТУРА}

1. Protosenya M.G., Karasev M.V., Belyakov N.A. (2015). Uprugo-plasticheskaya zadacha dlya vyrabotok razlichnyh form poperechnyh sechenij pri uslovii predelnogo sostoyaniya Kulona. Fiziko-texnicheskie problemy razrabotki poleznyh iskopaemyh (FTPRPI), 3, 48-56.

2. Vasyliev L.M., Vasyliev D.L. (2013). Teoreticheskoe obosnovanie formirovaniya gorizontalnyh normalnyh napryazhenij v massivah gornyh porod.FTPRPI,2,81-90.

3. Vasyliev L. M., Vasyliev D. L. (2015). Uchet kontaktnogo treniya $\mathrm{v}$ zadache o razrushenii gornyh porod szhatiem. FTPRPI, 3, 48-56.

4. Podyminogin G.M., Chanyshev A.I. (2015). Opredelenie maksimalno dopustimoj vysoty borta karera po sxeme zhestkoplasticheskogo tela. FTPRPI, 3, 32-40.

5. Nesmashnyj E.A., Bolotnikov A.V. (2017) .Opredelenie prochnosti skalnyh porod s ispol zovaniem sovremennogo oborudovaniya na primere mestorozhdeniya "Bolshaya Glivatka". Metallurgicheskaya i gornorudnaya promyshlennost, 3, 82-87.

6. Petrenko V.D., Tiutkin O.L., Lubinchyk O.I., Kildeev V. R. (2017). Rezultaty doslidzhennia stiikosti ukosiv zemlianoho polotna vysokykh nasypiv za dopomohoiu prohrami "OTKOS". Ukrainskaia zaliznytsa, 3-4 (45-46), 18-21.

7. Spravochnik (kadastr) fizicheskih svojstv gornyh porod. (1975). Pod red. Melnikova N.V., Rzhevskogo V.V., Protodyakonova M.M. Moskva: Nedra.

8. Baron L.I. Gorno-tehnicheskoe porodovedenie. (1977). M.: Nauka GOST 21153.2-88. Porodyi gornyie. Metodyi opredeleniya prochnost pri odnoosnom szhatii. (1984). M.: Goststandartizdat. 


\section{«Системні технології» 5 (130) 2020 «System technologies»}

\section{REFERENCES}

1. Protosenya M.G., Karasev M.V., Belyakov N.A. (2015). Uprugo-plasticheskaya zadacha dlya vyrabotok razlichnyh form poperechnyh sechenij pri uslovii predelnogo sostoyaniya Kulona. Fiziko-texnicheskie problemy razrabotki poleznyh iskopaemyh (FTPRPI), 3, 48-56.

2. Vasyliev L.M., Vasyliev D.L. (2013). Teoreticheskoe obosnovanie formirovaniya gorizontalnyh normalnyh napryazhenij v massivah gornyh porod.FTPRPI,2,81-90. 3. Vasyliev L.M., Vasyliev D.L. . (2015). Uchet kontaktnogo treniya v zadache o razrushenii gornyh porod szhatiem. FTPRPI, 3, 48-56.

4. Podyminogin, G.M., Chanyshev, A.I. (2015). Opredelenie maksimalno dopustimoj vysoty borta karera po sxeme zhestkoplasticheskogo tela. FTPRPI, 3, 32-40.

5. Nesmashnyj E.A., Bolotnikov A.V. (2017) .Opredelenie prochnosti skalnyh porod s ispol zovaniem sovremennogo oborudovaniya na primere mestorozhdeniya "Bolshaya Glivatka". Metallurgicheskaya i gornorudnaya promyshlennost, 3, 82-87.

6. Petrenko V.D., Tiutkin O.L., Lubinchyk O.I., Kildeev V. R. (2017). Rezultaty doslidzhennia stiikosti ukosiv zemlianoho polotna vysokykh nasypiv za dopomohoiu prohrami "OTKOS". Ukrainskaia zaliznytsa, 3-4 (45-46), 18-21.

7. Spravochnik (kadastr) fizicheskih svojstv gornyh porod. (1975). Pod red. Melnikov N.V., Rzhevskogo V.V., Protodyakonova M.M. Moskva: Nedra.

8. Baron L.I. Gorno-tehnicheskoe porodovedenie. (1977). M.: Nauka GOST 21153.2-88. Porodyi gornyie. Metodyi opredeleniya prochnost pri odnoosnom szhatii. (1984). M.: Goststandartizdat.

Received 13.03.2020. Accepted 16.03.2020.

Моделювання процесу руйнування циліндричних зразків гірських порід

Розроблено математичну модель руйнування циліндричних зразків гірничих порід при наявності контактного тертя. Розроблено метод розрахунку граничного вертикального напруження в вершині тріщини. і розрахунку межі міцності, в окремому випадку, циліндричних зразків гірських порід з використанням трьох показників властивостей (межа опору матеріалу зсуву, кут внутрішнього тертя, коефіцієнт контактного тертя), які простими способами можуть бути встановлені експериментально в умовах гірничорудних підприємств, де результати розрахунку можуть бути оперативно використані для управління процесами дезінтеграції.

Simulation of the process of destruction of cylindrical rock samples

The shear strength of a material is a constant for any point on the crack line. In this case, on the trajectory of maximum effective tangential stresses with the appearance of the first crack, spontaneous destruction of the body would occur. In real conditions, this does not happen. Layered rocks in the presence of cracks carry a certain load. The fact is that contact friction is inevitably involved in the fracture process and slip lines are not linear. During the destruction of brittle rocks with internal and external friction, the final result of the calculation of stresses at the crack tip in rock samples, and in general, in the massif, is significantly distorted. In the presence of contact tangential stresses, for example, from contact friction, the Coulomb criterion of the limiting state is met only locally, at the crack top. In other areas of the slip lines, the material is in an elastic state. 
«Системні технологіï» 5 (130) 2020 «System technologies»

Therefore, for layered rocks, this situation requires the development of a new approach to describing the process of local destruction, which must be taken into account.

The purpose of this article is to develop a mathematical model and reveal the mechanism of local rock destruction.

The work considers a cylindrical rock sample loaded with press plates with a distribution of contact stresses between the sample and the plate according to Prandtl.

A mathematical model is proposed and the mechanism of local fracture is revealed, which consists in the formation of ultimate shear strength of rocks at the crack tops - ultimate effective shear stresses and effective shear stress values that do not reach the shear strength at the points where their trajectories reach the boundary surface.

A dependence is derived for calculating the tensile strength of cylindrical rock samples with a truncated pyramidal form of fracture using the tensile strength of the material, the angle of internal friction, and the coefficient of contact friction, which can be easily established experimentally in mining enterprises, where the calculation results can be quickly used.

A comparison of the calculated values of the ultimate strength under uniaxial compression of the samples with the experimental ones convincingly indicates the high efficiency of the proposed analytical method.

Васильев Леонид Михайлович - д.т.н., профессор, Институт геотехнической механики им. Н.С. Полякова НАН Украины, Украина.

Васильев Дмитрий Леонидович - д.т.н., с.н.с, Институт геотехнической механики им. Н.С. Полякова НАН Украины, Украина.

Малич Николай Григорьевич - к.т.н., доцент, Национальная металлургическая академия Украины, Украина.

Назаров Александр Евгеньевич - м.н.с. Институт геотехнической механики им. Н.С. Полякова НАН Украины, Украина.

Васильєв Леонід Михайлович - д.т.н., професор, Інститут геотехнічної механіки ім. Н.С. Полякова НАН України, Україна.

Васильєв Дмитро Леонідович - д.т.н., с.н.с, Інститут геотехнічної механіки ім. Н.С. Полякова НАН України, Україна.

Маліч Микола Григорович - к.т.н., доцент, Національна металургійна академія України, Україна.

Назаров Олександр Євгенович - м.н.с. Інститут геотехнічної механіки ім. Н.С. Полякова НАН України, Україна.

Vasilyev Leonid - doctor of technical sciences, professor, Institute of Geotechnical Mechanics. N.S. Polyakov NAS of Ukraine, Ukraine.

Vasilyev Dmytro - doctor of technical sciences, doctor of science (Science), Institute of Geotechnical Mechanics. N.S. Polyakov NAS of Ukraine, Ukraine.

Malich Mykola - candidate of technical sciences, associate professor, $\mathrm{Na}$ tional Metallurgical Academy of Ukraine, Ukraine.

Nazarov Alexander - M.Sc. Institute of Geotechnical Mechanics. N.S. Polyakov NAS of Ukraine, Ukraine. 